\title{
DISCRETIZATION AND MOYAL BRACKETS
}

\section{ROBERT CARROLL}

\author{
(Received 7 March 2001)
}

\begin{abstract}
We give a $q$-analysis version of a discretization procedure of Kemmoku and Saito leading to an apparently new q-Moyal type bracket.
\end{abstract}

2000 Mathematics Subject Classification. 81R50, 81S30.

1. Introduction. We are pursuing further some of the directions spelled out in [4] relating Moyal-Weyl-Wigner theory, Hirota formulas, integrable systems, and discretization, with additional connections involving quantum groups (cf. $[1,2,3,6]$ ). In this paper, we indicate an apparently new $q$-Moyal type bracket formula arising in this context. In particular we follow here frameworks from [4, 18, 19, 20, 21, 22] for deformation quantization and integrable systems and refer to [1, 2, 3, 6] and references cited there for $q$-analysis and quantum groups. One objective will be to examine various formulas arising in the deformation of integrable systems and see if there are quantum group versions. Further we are looking for $q$-analysis versions of deformation quantization formulas in order to compare $q$-calculus and quantum group theory with deformation quantization. Thus for background one recalls for wave functions $\psi$ there are Wigner functions (WF) given via

$$
f(x, p)=\frac{1}{2 \pi} \int d y \psi^{*}\left(x-\frac{\hbar}{2} y\right) \exp (-i y p) \psi\left(x+\frac{\hbar}{2} y\right) .
$$

Then defining $f * g$ via

$$
\begin{gathered}
f * g=f \exp \left[\frac{i \hbar}{2}\left(\overleftarrow{\partial}_{x} \vec{\partial}_{p}-\overleftarrow{\partial}_{p} \vec{\partial}_{x}\right)\right] g \\
f(x, p) * g(x, p)=f\left(x+\frac{i \hbar}{2} \vec{\partial}_{p}, p-\frac{i \hbar}{2} \vec{\partial}_{x}\right) g(x, p)
\end{gathered}
$$

time dependence of WF's is given by $(H \sim$ Hamiltonian $)$

$$
\partial_{t} f(x, p, t)=\frac{1}{i \hbar}(H * f(x, p, t)-f(x, p, t) * H)=\{H, f\}_{M},
$$

where $\{f, g\}_{M} \sim$ Moyal bracket. As $\hbar \rightarrow 0$ this reduces to $\partial_{t} f-\{H, f\}=0$ (standard Poisson bracket). One can generalize and write out (1.2) in various ways. For example, replacing $i \hbar / 2$ by $\kappa$ one obtains as in [15]

$$
f * g=\sum_{0}^{\infty} \frac{\kappa^{s}}{s !} \sum_{j=0}^{s}(-1)^{j}\left(\begin{array}{l}
s \\
j
\end{array}\right)\left(\partial_{x}^{j} \partial_{p}^{s-j} f\right)\left(\partial_{x}^{s-j} \partial_{p}^{j} g\right)
$$


leading to $\left(\{f, g\}_{\kappa}=(f * g-g * f) / 2 \kappa\right)$

$$
\{f, g\}_{\kappa}=\sum_{0}^{\infty} \frac{\kappa^{2 s}}{(2 s+1) !} \sum_{j=0}^{2 s+1}(-1)^{j}\left(\begin{array}{c}
2 s+1 \\
j
\end{array}\right)\left(\partial_{x}^{j} \partial_{p}^{2 s+1-j} f\right)\left(\partial_{x}^{2 s+1-j} \partial_{p}^{j} g\right)
$$

(cf. also [29, 30, 31]) which will also be utilized in the form

$$
\begin{aligned}
f * g & =f e^{\kappa\left(\bar{\partial}_{x} \vec{\partial}_{p}-\bar{\partial}_{p} \vec{\partial}_{x}\right)} g \\
& =\left.e^{\left[\kappa\left(\partial_{x_{1}} \partial_{p_{2}}-\partial_{x_{2}} \partial_{p_{1}}\right)\right]} f\left(x_{1}, p_{1}\right) g\left(x_{2}, p_{2}\right)\right|_{(x, p)} \\
& =\sum_{0}^{\infty} \frac{(-1)^{r} \kappa^{r+s}}{r ! s !} \frac{\partial^{r+s} f}{\partial x^{r} \partial p^{s}} \frac{\partial^{r+s} g}{\partial p^{r} \partial x^{s}} \\
& =\sum_{0}^{\infty} \frac{\kappa^{n}(-1)^{n-s}}{s !(n-s) !}\left(\partial_{x}^{n-s} \partial_{p}^{s} f\right)\left(\partial_{x}^{s} \partial_{p}^{n-s} g\right) \\
& =\sum_{0}^{\infty} \frac{\kappa^{n}}{n !} \sum_{0}^{n}(-1)^{r}\left(\partial_{x}^{r} \partial_{p}^{n-r} f\right)\left(\partial_{x}^{n-r} \partial_{p}^{r} g\right)
\end{aligned}
$$

(note there are typos on page 169 in [4]) and for example one has

$$
g * f=g\left(x+\kappa \partial_{p}, p-\kappa \partial_{x}\right) f=f\left(x-\kappa \partial_{p}, p+\delta \partial_{x}\right) g .
$$

The Moyal bracket can then be defined via

$$
\begin{aligned}
\{f, g\}_{M} & =\frac{1}{\kappa}\left\{f \sin \left[\kappa\left(\overleftarrow{\partial}_{x} \vec{\partial}_{p}-\overleftarrow{\partial}_{p} \vec{\partial}_{x}\right)\right] g\right\} \\
& =\frac{1}{2 \kappa}(f * g-g * f) \\
& =\sum_{0}^{\infty} \frac{(-1)^{s} \kappa^{2 s}}{(2 s+1) !} \sum_{0}^{2 s+1}(-1)^{j}\left(\begin{array}{c}
2 s+1 \\
j
\end{array}\right)\left[\partial_{x}^{j} \partial_{p}^{2 s+1-j} f\right]\left[\partial_{x}^{2 s+1-j} \partial_{p}^{j} g\right]
\end{aligned}
$$

corresponding to $\kappa \rightarrow i \kappa$ in (1.5).

We emphasize also that many formulas in classical integrable systems already have a quantum mechanical (QM) flavor. For example in $[4,15,29,30,31]$ it was shown how there is a Moyal deformation $(K P)_{M}$ of dKP which for a particular value of $\kappa(\kappa=1 / 2$ in $[4,15])$ creates an equivalence $(K P)_{M} \equiv(K P)_{\text {Sato }}$. Actually QM features in integrable systems seem inevitable because of Lax operator formulations and the combinatorics inherent in Hirota equations and tau functions; also early work by the Kyoto school provided many connections between KP and quantum field theory (QFT) (cf. [7]). Such connections have since proliferated in topological field theory (TFT), Seiberg-Witten (SW) theory, and so forth, where for example, effective actions can correspond to tau functions of integrable systems and, somewhat paradoxically, effective slow dynamics or Whitham dynamics (obtained by averaging out fast fluctuations of angle variables) seems to correspond to a quantization (cf. [4, Chapter 5] or [5] for discussion). On the other hand, the so-called quantum inverse scattering method involving spin chains for quantum integrable systems (cf. [4, 12]), has a definite quantum group nature 
where the $R$-matrix provides quasitriangularity. The connection between $R$ and $r$ matrices leads one back to classical dynamics but the theories for two types of integrable systems (classical and quantum) have developed along different paths. It seems that various discretizations involving classical integrable systems (surveyed in [4]) should have a $q$-analysis foundation and thus there may be other forms of connecting glue between classical and quantum integrable systems via discretization.

2. Discretization and Moyal. In [4] we expanded at some length on a series of papers by Kemmoku, Saito, and collaborators (cf. [4] for references) and we now want to organize some of this in a better manner and develop matters somewhat further. Thus we sketch first some fundamental ideas. One defines

$$
\nabla=\frac{e^{\lambda \partial}-e^{-\lambda \partial}}{2 \lambda}=\frac{1}{\lambda} \sinh (\lambda \partial) ; \quad \nabla_{\mathbf{a}}=\frac{1}{\lambda} \sinh \left(\lambda \sum a_{i} \partial_{i}\right),
$$

where $a_{i} \sim \partial / \partial_{i}$ and $\partial \sim \partial_{x}$. Evidently (A1) $\nabla f(x)=(1 / 2 \lambda)[f(x+\lambda)-f(x-\lambda)]$ and $\nabla_{\mathbf{a}} f(\mathbf{x})=(1 / 2 \lambda)[f(\mathbf{x}+\mathbf{a})-f(\mathbf{x}-\mathbf{a})]$ (note that $\nabla_{\mathbf{a}}$ is not a vector). Then set (the $a_{i}$ correspond to unspecified local coordinates $x_{i}$ generating a lattice with vectors a in say $\mathbb{R}^{N}$ where $N \rightarrow \infty$ would require some convergence stipulations)

$$
X^{D}=\int d \mathbf{a} v_{\lambda}(\mathbf{x}, \mathbf{a}) \nabla_{\mathbf{a}} ; \quad \int d \mathbf{a} \sim \int \prod d a_{i} .
$$

Next a difference one form is defined via (A2) $\Omega_{D}=\int d \mathbf{a} w_{\lambda}(\mathbf{x}, \mathbf{a}) \Delta^{\mathbf{a}}$ where $\left\langle\Delta^{\mathbf{b}}, \nabla_{\mathbf{a}}\right\rangle=$ $\delta(\mathbf{b}-\mathbf{a})$ and $(\vec{a} \sim \mathbf{a})$

$$
\left\langle\Omega^{D}, X^{D}\right\rangle=\int d \vec{a} \int d \vec{b}\left\langle w_{\lambda}(\vec{x}, \vec{b}) \Delta^{\vec{b}}, v_{\lambda}(\vec{x}, \vec{a}) \nabla_{\vec{a}}\right\rangle=\int d \vec{a} w_{\lambda}(\vec{x}, \vec{a}) v_{\lambda}(\vec{x}, \vec{a}) .
$$

Note also $\Delta^{\mathbf{a}}$ can be realized via $\left(\left\langle\Delta^{\mathbf{a}}, \nabla_{\mathbf{b}}\right\rangle=\delta(\mathbf{a}-\mathbf{b})\right)$

$$
\Delta^{\mathrm{a}}=\lambda \operatorname{csch}[\lambda(\vec{a} \cdot \vec{\partial})]=\frac{2 \lambda}{e^{\lambda \vec{a} \cdot \vec{\partial}}-e^{-\lambda \vec{a} \cdot \vec{\partial}}}=2 \lambda \sum_{0}^{\infty} e^{-\lambda(2 n+1) \vec{a} \cdot \vec{\partial}} .
$$

In this connection we recall the $q^{2}$ difference operator (A3) $\partial_{q^{2}} f(x)=\left[f\left(q^{2} x\right)-f(x)\right] /$ $\left[\left(q^{2}-1\right) x\right]$ with "dual" a Jackson integral (A4) $\int_{0}^{y} d_{q^{2}} x f(x)=y\left(1-q^{2}\right) \sum_{0}^{\infty} f\left(y q^{2 n}\right) q^{2 n}$. According to $[19,20,21,22]$ there should be an unspecified $q$-analysis version of (2.4) related to pseudodifferential operators. We can develop an interesting $q$-analysis counterpart to (2.4) as follows. Note first that for $y=x+\lambda$ one can write (A5) $(1 / 2 \lambda)[f(x+$ $\lambda)-f(x-\lambda)]=[f(y+2 \lambda)-f(y)] / 2 \lambda$ and for $q^{2} y=y+2 \lambda$ one has $2 \lambda=\left(q^{2}-1\right) y$. Then consider (A6) $\tilde{\nabla}=[\exp (2 \lambda \partial)-1] / 2 \lambda$ with

$$
\begin{aligned}
\tilde{\nabla} f(y) & =\frac{f(y+2 \lambda)-f(y)}{2 \lambda}=\frac{f\left(q^{2} y\right)-f(y)}{\left(q^{2}-1\right) y}=\tilde{\partial}_{q^{2}} f(y) \\
& \equiv \tilde{\partial}_{q} f(z)=\frac{f(q z)-f\left(q^{-1} z\right)}{\left(q-q^{-1}\right) z}(q y=z),
\end{aligned}
$$

where $\tilde{\partial}_{q^{2}}$ involves now a variable $q=q(y)$ if $\lambda$ is to be regarded as constant (alternatively one could regard $\lambda$ as variable in $y$ and $q$ as constant or dispense with $\lambda$ 
altogether). For $\lambda$ constant (2.4) would become formally a $y$ dependent inverse (note $\left.\left(q^{2}-1\right) n y=2 n \lambda\right)$

$$
\tilde{\nabla}^{-1}=-2 \lambda\left(1-e^{2 \lambda \partial}\right)^{-1}=-2 \lambda \sum_{0}^{\infty} e^{2 n \lambda \partial}=\left(1-q^{2}\right) y \sum_{0}^{\infty} e^{\left(q^{2}-1\right) n y \partial}
$$

leading to

$$
\begin{aligned}
-2 \lambda\left(1-e^{2 \lambda \partial}\right)^{-1} g(y) & =G(y)=-2 \lambda \sum_{0}^{\infty} g(y+2 n \lambda) \\
& =\left(1-q^{2}\right) y \sum_{0}^{\infty} g\left(y+\left(q^{2}-1\right) n y\right) .
\end{aligned}
$$

Evidently (A7) $\tilde{\nabla} G(y)=g(y)$ so we can state (note a constant of integration in (2.7) would vanish for $\left.\int_{0}^{y} g \sim G(y)\right)$

Proposition 2.1. If we regard $q$ as $y$ dependent via $2 \lambda=\left(q^{2}-1\right) y$ with $\lambda$ constant then the inversion (2.7) has a modified Jackson type integral form

$$
\begin{aligned}
G(y) & =f_{0}^{y} g(x) d_{q^{2}} x \sim-2 \lambda\left(1-e^{2 \lambda \partial}\right)^{-1} g(y) \\
& =\left(1-q^{2}\right) y \sum_{0}^{\infty} g\left(y+\left(q^{2}-1\right) n y\right) .
\end{aligned}
$$

REMARK 2.2. Note $y$ is fixed throughout so the calculations make sense and this reveals also a property of Jackson integrals (A4), namely they do not seem to use the integration variable $x$ at all (although change of variable techniques should work). We emphasize that care is needed in using (2.5) in the form $\tilde{\partial}_{q^{2}}$ when computing $\tilde{\partial}_{q^{2}} G(y)=g(y)$. Thus $\tilde{\partial}_{q^{2}}$ defined via $\tilde{\nabla}$ in $(2.5)$ is not the same as $\partial_{q^{2}}$ unless provision is made for $\lambda=c$. If we try to compute $\partial_{q^{2}} G(y)$ without keeping $\lambda$ constant there arises an awkward term $\left(1-q^{2}\right) q^{2} y \sum_{0}^{\infty} g\left(q^{2} y+\left(q^{2}-1\right) n q^{2} y\right)$ and $\partial_{q^{2}} G(y) \neq g(y)$. The point is that $2 \lambda$ is constant and $\left(1-q^{2}\right) y=2 \lambda+\left(1-q^{2}\right) q^{2} y$. Nor does $y+2 n \lambda=$ $y+\left(1-q^{2}\right) n y$ go to $y+\left(1-q^{2}\right) n q^{2} y=y+2 n \lambda q^{2}$ (rather e.g., $y+2 n \lambda \rightarrow q^{2} y+2 n \lambda=$ $\left.y+2(n+1) \lambda=y+\left(1-q^{2}\right)(n+1) y\right)$. Thus for $\tilde{\partial}_{q^{2}} G(y)$ one must write $(1 / 2 \lambda)[G(y+$ $2 \lambda)-G(y)]=\left[\left(1-q^{2}\right) y\right]^{-1}\left[G\left(q^{2} y\right)-G(y)\right]=\partial_{q^{2}} G(y)$ as desired. If we regard this as a generally viable procedure of transferring "standard" differencing techniques in $\lambda$ to $q$-analysis then constant $\lambda$ steps for any $y$ correspond to constant steps $\left(1-q^{2}\right) y$ which means for large $y, q \rightarrow 1$, so if $G^{\prime}$ is continuous for example, then

$$
\tilde{\partial}_{q^{2}} G(y)=\frac{G\left(q^{2} y\right)-G(y)}{\left(q^{2}-1\right) y} \sim \frac{g(y+2 \lambda)-G(y)}{2 \lambda}=G^{\prime}(\xi)
$$

for $y \leq \xi \leq y+2 \lambda=q^{2} y$ and for $t$ large $y+2 \lambda \simeq y$ corresponds to $q^{2} \rightarrow 1$. There seems to be no reason not to use the $q, \lambda$ correspondence in general as long as computational consistency is maintained.

REMARK 2.3. We will eventually dispense with $\lambda$ altogether in rephrasing matters entirely in $q$ so that $\tilde{\partial}_{q^{2}}$ or $\tilde{\partial}_{q}$ will not arise. 
Continuing now from [4] one can define difference 2-forms $\Omega_{2}^{D}$, an exterior difference operator $\Delta$, and a Lie difference operator via (standard $\wedge$ product)

$$
\begin{aligned}
\Omega_{2}^{D} & =\int d \mathbf{a} \int d \mathbf{b} w_{\lambda}(\mathbf{x}, \mathbf{a}, \mathbf{b}) \Delta^{\mathbf{a}} \wedge \Delta^{\mathbf{b}} ; \\
\Delta \Omega_{2}^{D} & =\int d \mathbf{a} \int d \mathbf{b} \int d \mathbf{c} \nabla_{\mathbf{a}} w_{\lambda}(\mathbf{x}, \mathbf{a}, \mathbf{b}) \Delta^{\mathbf{c}} \wedge \Delta^{\mathbf{a}} \wedge \Delta^{\mathbf{b}} .
\end{aligned}
$$

Since $\left[\nabla_{\mathbf{a}}, \nabla_{\mathbf{b}}\right]=0$ one has $\Delta \Delta=0$ and finally for $X^{D}$ as in (2.2)

$$
i_{\nabla_{\mathbf{c}}}\left(\Delta^{\mathbf{a}} \wedge \Delta^{\mathbf{b}}\right)=\delta(\mathbf{c}-\mathbf{a}) \Delta^{\mathbf{b}}-\delta(\mathbf{c}-\mathbf{b}) \Delta^{\mathbf{a}} ; \quad \hat{\Sigma}_{X^{D}}=\Delta \cdot i_{X^{D}}+i_{X^{D}} \cdot \Delta .
$$

Now consider a phase space $\vec{x} \sim \mathbf{x}=(x, p)$ and in place of (A8) $X_{f} \mathcal{g}=\left(f_{p} \partial_{x}-f_{x} \partial_{p}\right) \mathcal{g}$ one writes (A9) $X_{f}^{D}=\int d a_{1} d a_{2} v_{\lambda}[f]\left(x, p, a_{1}, a_{2}\right) \nabla_{\mathbf{a}}$ where (cf. (2.2))

$$
\begin{aligned}
& v_{\lambda}[f]\left(x, p, a_{1}, a_{2}\right) \\
& \quad=\left(\frac{\lambda}{2 \pi}\right)^{2} \int d b_{1} d b_{2} \exp \left[-i \lambda\left(a_{1} b_{2}-a_{2} b_{1}\right)\right] f\left(x+\lambda b_{1}, p+\lambda b_{2}\right)
\end{aligned}
$$

which should correspond to $\left\langle\Delta^{\mathrm{a}}, X_{f}^{D}\right\rangle$ (cf. Section 3). Note $a_{1} b_{2}-a_{2} b_{1}$ can be written as $\vec{a} \times \vec{b}$ and $(1 / \lambda)(\vec{a} \times \vec{b})$ is the area in $\lambda$ units of the parallelogram formed by $\vec{a} \times \vec{b}(\lambda$ is essentially a scaling factor here and not a Fourier variable). The symplectic structure of (A8) is retained via an interchange of $\vec{a}$ and $\vec{b}$. We note that (A9) can be written in the form (the details are in [4])

$$
\begin{aligned}
X_{f}^{D}=-\frac{i \lambda}{(2 \pi)^{2}} \int d a_{1} d a_{2} \int & d b_{1} d b_{2} \sin \left[\lambda\left(a_{1} b_{2}-a_{2} b_{1}\right)\right] \\
& \times f\left(x+\lambda b_{1}, p+\lambda b_{2}\right) e^{\lambda\left(a_{1} \partial_{x}+a_{2} \partial_{p}\right)}
\end{aligned}
$$

leading to

$$
\begin{gathered}
X_{f}^{D} g=-\frac{i \lambda}{(2 \pi)^{2}} \iint d a_{1} d a_{2} \iint d b_{1} d b_{2} \sin \left[\lambda\left(a_{1} b_{2}-a_{2} b_{1}\right)\right] f\left(x+\lambda b_{1}, p+\lambda b_{2}\right) \\
\times \exp \left[\lambda\left(a_{1} \partial_{x}+a_{2} \partial_{p}\right)\right] g(x, p) \\
=-\frac{i \lambda}{(2 \pi)^{2}} \int d a \int d b \sin \left[\lambda\left(a_{1} b_{2}-a_{2} b_{1}\right)\right] f\left(x+\lambda b_{1}, p+\lambda b_{2}\right) \\
\times g\left(x+\lambda a_{1}, p+\lambda a_{2}\right) .
\end{gathered}
$$

Subsequent calculation gives, using $x+\lambda a_{1}=\alpha_{1}$ and $p+\lambda a_{2}=\alpha_{2}$ (cf. [4])

$$
\begin{aligned}
\int d a & \int d b e^{i \lambda\left(a_{1} b_{2}-a_{2} b_{1}\right)} f\left(x+\lambda b_{1}, p+\lambda b_{2}\right) g\left(x+\lambda a_{1}, p+\lambda a_{2}\right) \\
& =\frac{1}{\lambda^{2}}\left(\int f\left(x+i \lambda \partial_{\alpha_{2}}, p-i \lambda \partial_{\alpha_{1}}\right) \int e^{i\left[b_{2}\left(\alpha_{1}-x\right)-b_{1}\left(\alpha_{2}-p\right)\right]} d b\right) g\left(\alpha_{1}, \alpha_{2}\right) d \alpha \\
& =\left(\frac{2 \pi}{\lambda}\right)^{2} f\left(x-i \lambda \partial_{p}, p+i \lambda \partial_{x}\right) g(x, p) \sim\left(\frac{2 \pi}{\lambda}\right)^{2} g * f
\end{aligned}
$$

leading finally to

$$
X_{f}^{D} g=\left.\frac{i}{\lambda} \sin \left[\lambda\left(\partial_{x_{1}} \partial_{p_{2}}-\partial_{p_{1}} \partial_{x_{2}}\right)\right] f\left(p_{1}, x_{1}\right) g\left(p_{2}, x_{2}\right)\right|_{(p, x)}=\{f, g\}_{M}
$$


In addition, from the Jacobi identity for the Moyal bracket one has

$$
\begin{aligned}
{\left[X_{f}^{D}, X_{g}^{D}\right] h } & =X_{f}^{D}\{g, h\}-X_{g}^{D}\{f, h\}=\{f,\{g, h\}\}-\{g,\{f, h\}\} \\
& =\{\{f, g\}, h\}=X_{\{f, g\}}^{D} h .
\end{aligned}
$$

A symplectic form can also be given via

$$
\Omega=\frac{1}{2 \lambda} \iint d a_{1} d a_{2} \iint d b_{1} d b_{2} e^{i \lambda\left(a_{1} b_{2}-a_{2} b_{1}\right)} \Delta^{\mathbf{a}} \wedge \Delta^{\mathbf{b}}
$$

and this satisfies $i_{X_{f}^{D}} \Omega=\Delta f$ (analogous to $i_{X_{f}} \omega=d \omega$ for a symplectic form $\omega$ ). Our formulas differ at times by $\pm i$ from $[18,19,20,21,22]$ but everything seems consistent and correct here; the philosophy of running $a_{i}$ over $\mathbb{R} \sim(-\infty, \infty)$ is crucial in the calculations (alternatively $\int$ could represent a sum over a discrete symmetric set, for example, $[-N, N]$ with $N$ infinite or not). We note also a somewhat quasi Fourier theoretic version of the formulas (A9), (2.12), (2.13), and so forth, developed in [4]. Thus consider

$$
v_{\lambda}[f](\mathbf{x}, \mathbf{a})=\left(\frac{\lambda}{2 \pi}\right)^{2} \int d \mathbf{b} e^{-i \lambda(\mathbf{a} \times \mathbf{b})} e^{\lambda \vec{b} \cdot \vec{\partial}} f
$$

Hence (using $\mathbf{b} \rightarrow-\mathbf{b}$ )

$$
v_{\lambda}[f](\mathbf{x},-\mathbf{a})=\left(\frac{\lambda}{2 \pi}\right)^{2} \int d \mathbf{b} e^{i \lambda(\mathbf{a} \times \mathbf{b})} e^{\lambda \vec{b} \cdot \vec{\partial}} f=\left(\frac{\lambda}{2 \pi}\right)^{2} \int d \mathbf{b} e^{-i \lambda(\mathbf{a} \times \mathbf{b})} e^{-\lambda \vec{b} \cdot \vec{\partial}} f
$$

and since $\nabla_{-\mathbf{a}}=-\nabla_{\mathbf{a}}$ one gets

$$
X_{f}^{D}=\int d \mathbf{a} v_{\lambda}[f](\mathbf{x}, \mathbf{a}) \nabla_{\mathbf{a}}=-\int_{\infty}^{-\infty} d \mathbf{a} v_{\lambda}[f](\mathbf{x},-\mathbf{a}) \nabla_{-\mathbf{a}}=-\int d \mathbf{a} v_{\lambda}[f](\mathbf{x},-\mathbf{a}) \nabla_{\mathbf{a}} .
$$

Consequently,

$$
\begin{aligned}
X_{f}^{D} & =\frac{1}{2} \int d \mathbf{a}\left[v_{\lambda}[f](\mathbf{x}, \mathbf{a})-v_{\lambda}[f](\mathbf{x},-\mathbf{a})\right] \nabla_{\mathbf{a}} \\
& =\frac{\lambda^{3}}{4 \pi^{2}} \int d \mathbf{a} \int d \mathbf{b} e^{-i \lambda(\mathbf{a} \times \mathbf{b})}\left\{\frac{e^{\lambda \vec{b} \cdot \vec{\partial}}-e^{-\lambda \vec{b} \cdot \vec{\partial}}}{2 \lambda}\right\} f \nabla_{\mathbf{a}} \\
& =\frac{\lambda^{3}}{4 \pi^{2}} \int d \mathbf{a} \int d \mathbf{b} e^{-i \lambda(\mathbf{a} \times \mathbf{b})} \nabla_{\mathbf{b}} f \nabla_{\mathbf{a}} .
\end{aligned}
$$

This formula provides another representation for $X_{f}^{D}$ via

$$
X_{f}^{D}=\int d \mathbf{a} \tilde{v}_{\lambda}[f](\mathbf{x}, \mathbf{a}) \nabla_{\mathbf{a}} ; \quad \tilde{v}_{\lambda}[f](\mathbf{x}, \mathbf{a})=\frac{\lambda^{3}}{4 \pi^{2}} \int d \mathbf{b} e^{-i \lambda(\mathbf{a} \times \mathbf{b})} \nabla_{\mathbf{b}} f .
$$

The above gives a direct discretization of phase space and the natural difference analogue of Lie bracket leads to the Moyal bracket. Thus one takes $\lambda \sim \hbar / 2$ and defines $X_{A}^{Q}=\hbar X_{A}^{D}$ for functions $A(x, p)$ and there is a Heisenberg equation $(H \sim$ Hamiltonian $)$ (A10) $i \hbar \partial_{t} X_{A}^{Q}=\left[X_{A}^{Q}, X_{H}^{Q}\right]$ (where both $A$ and $H$ may contain $\hbar$ ). This is compatible with (A11) $\partial_{t} A=\{A, H\}_{M}$ (cf. (2.16) and (2.17)). To see how this works we recall the standard quantum mechanical (QM) idea of Wigner distribution function $F_{w}$ with $\int F_{w} d x=$ 1 and $\langle\hat{A}\rangle=\int F_{w} A d x$ for the expectation value of an operator $\hat{A}$ associated to the 
observable function $A$ (Weyl ordering is to be invoked when ordering is needed and details are in [4]). The corresponding discrete version is given via a difference 1-form

$$
P_{F_{w}}=\frac{\hbar}{4} \iint d a_{1} d a_{2} \iint d b_{1} d b_{2} e^{i \hbar\left(a_{1} b_{2}-a_{2} b_{1}\right) / 2} F_{w}\left(x+\frac{\hbar}{2} b_{1}, p+\frac{\hbar}{2} b_{2}\right) \Delta^{a}
$$

so (A12) $\left\langle P_{F_{w}}, X_{A}^{Q}\right\rangle=\int d x d p F_{w}(x, p) A(x, p)=\langle\hat{A}\rangle$. In the Heisenberg picture the time dependence is (A13) $\partial_{t}\left\langle P_{F_{w}}, X_{A}^{Q}\right\rangle=\left\langle P_{F_{w}}, X_{A}^{Q}(t)\right\rangle$ which in the Schrödinger picture becomes (A14) $\partial_{t}\left\langle P_{F_{w}}, X_{A}^{Q}\right\rangle=\left\langle P_{F_{w}}(t), X_{A}^{Q}\right\rangle$. Here the solution of (A10) necessarily is

$$
X_{A}^{Q}(t)=\exp \left(-\frac{i t}{\hbar} X_{H}^{Q}\right) X_{A}^{Q} \exp \left(\frac{i t}{\hbar} X_{H}^{Q}\right)
$$

(simply differentiate $X_{A}^{Q}=\exp \left[(i t / \hbar) X_{H}^{Q}\right] X_{A}^{Q}(t) \exp \left[(-i t / \hbar) X_{H}^{Q}\right]$ and note that in (A10) $X_{A}^{Q} \sim X_{A}^{Q}(t)$ ). This corresponds to a solution of (A11) of the form (A15) $A(t)=$ $\left[\exp (i t / \hbar) X_{H}^{Q}\right] A$ and in the Heisenberg picture

$$
-i \hbar \frac{d}{d t}\left\langle P_{F_{w}}, X_{A}^{Q}(t)\right\rangle=\left\langle P_{F_{w}},\left[X_{A}^{Q}(t), X_{H}^{Q}\right]\right\rangle=\left\langle P_{F_{w}}, X_{\{A(t), H\}_{M}}^{Q}\right\rangle,
$$

where the right side is $\left\langle P_{\left\{H, F_{w}(t)\right\}_{M}}, X_{A}^{Q}\right\rangle$ upon defining (A16) $F_{w}(t)=\exp \left[-(i t / \hbar) X_{H}^{Q}\right] F_{w}$ so that (A17) $\partial_{t} P_{F_{w}(t)}=P_{\left\{H, F_{w}(t)\right\}_{M}} \equiv \partial_{t} F_{w}(t)=\left\{H, F_{w}(t)\right\}_{M}$.

3. $Q$-discretization. Consider now a variation on Section 2 based on a $q$-lattice. This will constitute a different approach from those in Remark 2.2 and Proposition 2.1 in that we keep $q$ fixed. Indeed $q$ (or $f(q)$ ) can play the role of $\lambda$ and we write

$$
\begin{aligned}
\hat{\nabla}_{m n} f(x, p) & =\frac{f\left(x q^{2 m}, p q^{2 n}\right)-f(x, p)}{\left(q^{2 m}-1\right) x\left(q^{2 n}-1\right) p}, \\
\check{\nabla}_{m n} g(x, p) & =\frac{g\left(x q^{m}, p q^{n}\right)-g\left(x q^{-m}, p q^{-n}\right)}{\left(q^{m}-q^{-m}\right)\left(q^{n}-q^{-n}\right) x p} \\
& =\frac{e^{\lambda(m, n) \cdot\left(\hat{\partial}_{1}, \hat{\partial}_{2}\right)}-e^{-\lambda(m, n) \cdot\left(\hat{\partial}_{1}, \hat{\partial}_{2}\right)}}{\left(q^{m}-q^{-m}\right)\left(q^{n}-q^{-n}\right) x p} \hat{g}(\log (x), \log (p)) \\
& =q^{m+n} e^{-\lambda(m, n) \cdot\left(\hat{\partial}_{1}, \hat{\partial}_{2}\right)} \hat{\nabla}_{m n} \mathcal{g}=G,
\end{aligned}
$$

so $(m, n)$ plays the role of Fourier variables $\left(a_{1}, a_{2}\right) \sim$ a. We recall from [4] the device (A18) $\lambda=\log (q), \exp (\lambda)=q, f(x)=\hat{f}(\log (x)), q^{2 m x \partial_{x}} f(x)=\exp \left[2 m \lambda \partial_{\log (x)}\right] \hat{f}(\log (x))$ $=\hat{f}(\log (x)+2 m \log (q))=\hat{f}\left(\log \left(q^{2 m} x\right)\right)=f\left(x q^{2 m}\right)$. This suggests an inversion for $\hat{\nabla}_{m n}$ written via

$$
\hat{\nabla}_{m n} f(x, p)=\frac{\left(e^{2 \lambda(m, n) \cdot\left(\hat{\partial}_{1}, \hat{\partial}_{2}\right)}-1\right)}{\left(q^{2 m}-1\right) x\left(q^{2 n}-1\right) p} \hat{f}(\log (x), \log (p))
$$

$\left(\hat{\partial}_{1}=\partial / \partial \log (x), \hat{\partial}_{2}=\partial / \partial \log (p)\right)$ in a form similar to a Jackson integral. Thus first we 
can derive a Jackson integral as follows. Write

$$
\nabla f(x)=\partial_{q^{2}} f(x)=\frac{f\left(q^{2} x\right)-f(x)}{\left(q^{2}-1\right) x}=\frac{\left(e^{2 \lambda x \partial x}-1\right)}{\left(q^{2}-1\right) x} f(x)=g(x)
$$

with formally

$$
f(x)=\left(1-q^{2}\right) \sum_{0}^{\infty} e^{2 k \lambda x \partial_{x}}(x g(x))=\left(1-q^{2}\right) \sum_{0}^{\infty} q^{2 k} x g\left(q^{2 k} x\right)
$$

which is the Jackson integral $\int_{0}^{x} d_{q^{2}} y g(y)$. Similarly, we can write now formally

$$
\begin{aligned}
\hat{\nabla}_{m n}^{-1} g(x, p) & =-\left(q^{2 m}-1\right)\left(q^{2 n}-1\right) \sum_{0}^{\infty} e^{2 \lambda k(m, n) \cdot\left(\hat{\partial}_{1}, \hat{\partial}_{2}\right)}(x p g(x, p)) \\
& =-\left(q^{2 m}-1\right)\left(q^{2 n}-1\right) \sum_{0}^{\infty} q^{2 m k} x q^{2 n k} p g\left(q^{2 m k} x, q^{2 n k} p\right) \\
& =G(x, p)
\end{aligned}
$$

This can be checked via

$$
\begin{aligned}
\frac{G\left(q^{2 m} x, q^{2 n} p\right)-G(x, p)}{\left(q^{2 m}-1\right) x\left(q^{2 n}-1\right) p}= & g(x, p) \\
= & -\sum_{0}^{\infty} q^{2 m(k+1)} q^{2 n(k+1)} g\left(q^{2 m(k+1)} x, q^{2 n(k+1)} p\right) \\
& +\sum_{0}^{\infty} q^{2 m k} q^{2 n k} g\left(q^{2 m k} x, q^{2 n k} p\right)
\end{aligned}
$$

Hence we have proved

Proposition 3.1. The difference operator $\hat{\nabla}_{m n}$ of (3.1) can be inverted via (3.6) as a kind of extended Jackson integral. Similarly one has

$$
\begin{aligned}
\check{\nabla}_{m n}^{-1} g(x, p)= & q^{-m-n} \hat{\nabla}_{m n}^{-1} g\left(x q^{-m}, p q^{-n}\right) \\
= & -q^{-m-n}\left(q^{2 m}-1\right)\left(q^{2 n}-1\right) \\
& \times \sum_{0}^{\infty} q^{2 m k-m} x q^{2 n k-n} p g\left(q^{2 m k-m} x, q^{2 n k-n} p\right) \\
= & -\left(q^{m}-q^{-m}\right)\left(q^{n}-q^{-n}\right) x p \\
& \times \sum_{0}^{\infty} q^{(2 k-1)(m+n)} g\left(q^{(2 k-1) m} x, q^{(2 k-1) n} p\right) .
\end{aligned}
$$

It should be possible now to duplicate most of the machinery in Section 2 with $q$ discretization as above. We note that this procedure and the resulting formulas appear to be different from any of the phase space discretizations in $[8,10,13,16,23,24$, $25,26,27,28,32,33]$. We will consider an analogue of $X_{f}^{D}$ in (A9) or (2.23) via

$$
\hat{X}_{f}^{D}=\sum_{m, n} v_{q}[f](x, p, m, n) \hat{\nabla}_{m n} \quad \text { or } \quad \check{X}_{f}^{D}=\sum v_{q}[f](x, p, m, n) \check{\nabla}_{m n},
$$

where we need then a formula for $v_{q}[f]$ which can perhaps be modeled on (2.23) in 
a quasi-Fourier spirit. Note that the stipulation $\left\langle\Delta^{\mathbf{a}}, \nabla_{\mathbf{b}}\right\rangle=\delta(\mathbf{a}-\mathbf{b})$, or $\hat{\Delta}^{m n}=\hat{\nabla}_{m n}^{-1}$ as in (3.6) and (3.7) simply provides a tautology (A19) $v_{q}[f](x, p, m, n)=\left\langle\hat{\Delta}^{m n}, \hat{X}_{f}^{D}\right\rangle$ or as in (2.12) the equation (A20) $\left\langle\Delta^{\mathbf{a}}, X_{f}^{D}\right\rangle=\left\langle\Delta^{\mathbf{a}}, \int d \mathbf{b} v_{\lambda}[f](x, p, \mathbf{b}) \nabla_{\mathbf{b}}\right\rangle=v_{\mathbf{a}}[f](x, p, \mathbf{a})$. Thus one should realize that $v_{\lambda}[f]$ is simply selected in an ad hoc manner so that $X_{f}^{D} \mathcal{g}=\{f, g\}_{M}$. It turns out that the use of $\hat{\nabla}_{m n}$ and $\hat{X}_{f}^{D}$ would not reproduce a suitable \pm symmetry for a quasi-Fourier approach so we will concentrate on $\breve{X}_{f}^{D}$ and $\check{\nabla}_{m n}$.

In [9] a quantum $q$-Moyal bracket $(\hbar \neq 0)$ is suggested in the form

$$
\left\{p^{m} x^{n}, p^{k} x^{\ell}\right\}_{q M}=\frac{1}{i \hbar}\left(q^{n k} p^{m} x^{n} * p^{k} x^{\ell}-q^{m \ell} p^{k} x^{\ell} * p^{m} x^{n}\right),
$$

where $*$ can refer to standard or antistandard orderings via $\left(v=\log (q)\right.$ and $\left.D_{z} \sim \partial_{q}\right)$

$$
\begin{aligned}
& *_{s} \equiv \sum_{0}^{\infty} \frac{(i \hbar)^{r}}{[r] !} \overleftarrow{D}_{p}^{r} \exp \left(v \overleftarrow{\partial}_{p} p x \vec{\partial}_{x}\right) \vec{D}_{x}^{r} \\
& *_{A} \equiv \sum_{s=0}^{\infty}\left(-v \overleftarrow{\partial}_{x} x\right)^{s} \sum_{r=0}^{s} \frac{(-i \hbar)^{r} q^{r(r-1) / 2}}{[r] !} \overleftarrow{D}_{x}^{r} \vec{D}_{p}^{r}\left(p \vec{\partial}_{p}\right)^{s}
\end{aligned}
$$

Here standard ordering involves XP products and antistandard has PX products (see Section 4). The symbol map is $S_{S}\left(X^{m} P^{n}\right)=S_{A}\left(P^{m} X^{n}\right)=p^{m} x^{n}$; Weyl ordering is also considered but there are some complications. We note also for $\hbar=0$ one has classical star products based on $(v=\log (q)-$ cf. $[9,11])$

$$
\begin{aligned}
& *_{S}^{q} \equiv \exp \left(v \overleftarrow{\partial}_{p} p x \vec{\partial}_{x}\right) \\
& *_{A}^{q} \equiv \exp \left(-v \overleftarrow{\partial}_{x} x p \vec{\partial}_{p}\right) \\
& *_{W}^{q} \equiv \exp \left(-\frac{v}{2}\left(\overleftarrow{\partial}_{x} x p \vec{\partial}_{p}-\overleftarrow{\partial}_{p} p x \vec{\partial}_{x}\right)\right)
\end{aligned}
$$

(here $*_{W}^{q}$ refers to Weyl ordering); these star products all satisfy

$$
q^{n k} p^{m} x^{n} *^{q} p^{k} x^{\ell}-q^{m \ell} p^{k} x^{\ell} *^{q} p^{m} x^{n}=0
$$

as required for standard $q$-deformed operators.

4. Calculations. For completeness we will give a number of calculations to show how our results are parallel to Section 2 and can be reached through some quasiFourier type procedures. First we recall some useful formulas (cf. [7, 14, 17]), namely

$$
\delta(z-w)=z^{-1} \sum_{n \in \mathbf{Z}}\left(\frac{z}{w}\right)^{n}=z^{-1} \hat{\delta}\left(\frac{q}{w}\right) .
$$

There are many nice calculations available using (4.1); we mention for example, $\left(\operatorname{Res}_{z} \sum a_{n} z^{n}=a_{-1}\right.$ and $\left.D_{z}=z(d / d z)\right)$

$$
\begin{gathered}
\delta(w-z)=w^{-1} \sum_{\mathrm{Z}}\left(\frac{w}{z}\right)=w^{-1} \sum_{\mathrm{Z}}\left(\frac{z}{w}\right)^{n}=z^{-1} \sum\left(\frac{z}{w}\right)^{n}=\delta(z-w) \\
\operatorname{Res}_{z} f(z) \delta(z-w)=f(w) ; \quad f(z) \hat{\delta}(a z)=f\left(a^{-1}\right) \hat{\delta}(a z) \\
\operatorname{Res}_{z} \partial a(z) b(z)=-\operatorname{Res}_{z} a(z) \partial b(z)
\end{gathered}
$$


This will provide a delta function corresponding to $\int \exp \left[i b_{2}\left(\alpha_{1}-x\right)-i b_{1}\left(\alpha_{2}-p\right)\right] d \mathbf{b}$. Now, leaving aside possible multiplicative factors (cf. Remark 4.4), consider (2.12) in the form

$$
v_{q}[f](x, p, \mathbf{a})=c(q) \sum_{r, s} q^{m s-n r} f\left(q^{r} x, q^{s} p\right)
$$

leading to (cf. (2.13) and (2.14))

$$
\begin{aligned}
X_{f}^{D} & =\hat{c}(q) \sum_{m, n, r, s}\left(q^{m s-n r}-q^{-m s+n r}\right) f\left(q^{r} x, q^{s} p\right) \cdot q^{(m, n) \cdot\left(\hat{\partial}_{1}, \hat{\partial}_{2}\right)} ; \\
X_{f}^{D} \mathcal{g} & =\hat{c}(q) \sum_{m, n, r, s}\left(q^{m s-n r}-q^{-m s+n r}\right) f\left(q^{r} x, q^{s} p,\right) g\left(q^{m} x, q^{n} p\right)
\end{aligned}
$$

while (2.15) can be written as $\left(x+\lambda a_{1}=\alpha_{1}\right.$ and $\left.p+\lambda a_{2}=\alpha_{2}\right)$

$$
\begin{aligned}
\int d a \int & d b e^{i \lambda\left(a_{1} b_{2}-a_{2} b_{1}\right)} f\left(x+\lambda b_{1}, p+\lambda b_{2}\right) g\left(x+\lambda a_{1}, p+\lambda a_{2}\right) \\
& \times \frac{1}{\lambda^{2}} \iint d \alpha d b e^{i\left[b_{2}\left(\alpha_{1}-x\right)-b_{1}\left(\alpha_{2}-p\right)\right]} f\left(x+\lambda b_{1}, p+\lambda b_{2}\right) g\left(\alpha_{1}, \alpha_{2}\right) \\
= & \frac{1}{\lambda^{2}}\left(\int f\left(x+i \lambda \partial_{\alpha_{2}}, p-i \lambda \partial_{\alpha_{1}}\right) \int e^{i\left[b_{2}\left(\alpha_{1}-x\right)-b_{1}\left(\alpha_{2}-p\right)\right]} d b\right) g\left(\alpha_{1}, \alpha_{2}\right) d \alpha \\
= & \left(\frac{2 \pi}{\lambda}\right)^{2} \int\left[f\left(x+i \lambda \partial_{\alpha_{2}}, p-i \lambda \partial_{\alpha_{1}}\right) \delta\left(\alpha_{1}-x, \alpha_{2}-p\right)\right] g\left(\alpha_{1}, \alpha_{2}\right) d \alpha \\
= & \left(\frac{2 \pi}{\lambda}\right)^{2} f\left(x-i \lambda \partial_{p}, p+i \lambda \partial_{x}\right) g(x, p) \sim\left(\frac{2 \pi}{\lambda}\right)^{2} g * f .
\end{aligned}
$$

Intuitively one thinks of $\lambda \sim \log (q), \mathbf{a} \sim(m, n)$, and $\mathbf{b} \sim(r, s)$ so the substitution $x+\lambda a_{1}=\alpha_{1}$ corresponds to $\alpha_{1} / x=q^{m}$; similarly $\alpha_{2} / p=q^{n}$ and the second and third lines in (4.5) correspond to

$$
\Gamma_{1}=c(q, p, x) \sum_{\alpha} \sum_{r, s}\left(\frac{\alpha_{1}}{x}\right)^{s}\left(\frac{\alpha_{2}}{p}\right)^{r} f\left(x q^{r}, p q^{s}\right) g\left(\alpha_{1}, \alpha_{2}\right),
$$

where $\sum_{\alpha} \sim \operatorname{Res}_{\alpha}\left(1 / \alpha_{1} \alpha_{2}\right)$. The first question is to ask if we can write something like

$$
\sum_{r, s} f\left(x q^{r}, p q^{s}\right)\left(\frac{\alpha_{1}}{x}\right)^{s}\left(\frac{\alpha_{2}}{p}\right)^{-r} \sim f\left(x q^{\hat{\partial}_{1}}, p q^{-\hat{\partial}_{2}}\right) \hat{\delta}\left(\frac{\alpha_{1}}{x}\right) \hat{\delta}\left(\frac{p}{\alpha_{2}}\right)
$$

in analogy to lines 3 and 4 of (4.5). We could imagine for example, $f(x, p)=\sum a_{k \ell} x^{k} p^{\ell}$ and look at

$$
\begin{aligned}
\sum_{r, s} x^{k} p^{\ell} q^{k r} q^{\ell s}\left(\frac{\alpha_{1}}{x}\right)^{s}\left(\frac{\alpha_{2}}{p}\right)^{-r} & =\sum_{r, s} x^{k} p^{\ell} q^{-k \hat{\partial}_{2}} q^{\ell \hat{\partial}_{1}}\left(\frac{\alpha_{1}}{x}\right)^{s}\left(\frac{\alpha_{2}}{p}\right)^{-r} \\
& =x^{k} p^{\ell} q^{-k \hat{\partial}_{2}} q^{\ell \hat{\partial}_{1}} \hat{\delta}\left(\frac{\alpha_{1}}{x}\right) \hat{\delta}\left(\frac{p}{\alpha_{2}}\right)
\end{aligned}
$$

since $q^{-k \hat{\partial}_{2}}\left(\alpha_{2} / p\right)^{-r}=\left(q^{-k} \alpha_{2} / p\right)^{-r}=q^{k r}\left(\alpha_{2} / p\right)^{r}$. Consequently, for $f=\sum a_{k \ell} x^{k} p^{\ell}$ 
in (4.6) we have

$$
\Gamma_{1}=c(q, p, x) \sum_{k, \ell} a_{k \ell} x^{k} p^{\ell} q^{-k p \partial_{p}} q^{\ell x \partial_{x}} g(x, p)
$$

since $\operatorname{Res}_{\alpha}\left(1 / \alpha_{1} \alpha_{2}\right) \hat{\delta}\left(\alpha_{1} / x\right) \hat{\delta}\left(p / \alpha_{2}\right) g\left(\alpha_{1}, \alpha_{2}\right)=g(x, p)$ and for example, $\hat{\partial}_{1}$ in $\alpha_{1}$ becomes $\hat{\partial}_{1}=x \partial_{x}$. This leads to

$$
\Gamma_{1}=c(q, p, x) \sum a_{k \ell} x^{k} p^{\ell} g\left(x q^{\ell}, p q^{-k}\right)
$$

as a putative $g * f(\mathrm{cf} .(4.5))$. For $g=\sum b_{\gamma \beta} x^{\gamma} p^{\beta}$ this corresponds to

$$
\Gamma_{1}(f, g)=c \sum_{k, \ell, \gamma \beta} a_{k \ell} b_{\gamma \beta} x^{k+\gamma} p^{\ell+\beta} q^{\ell \gamma-k \beta} \sim g * f .
$$

The terms of the form (2.15) corresponding to $\exp \left[-i \lambda\left(a_{1} b_{2}-a_{2} b_{1}\right)\right]$ in (2.14) involve now in place of (4.7) a term

$$
-\sum_{r, s} f\left(x q^{r}, p q^{s}\right)\left(\frac{\alpha_{1}}{x}\right)^{-s}\left(\frac{\alpha_{2}}{p}\right)^{r}=-f\left(x q^{-\hat{\partial}_{1}}, p q^{\hat{\partial}_{2}}\right) \hat{\delta}\left(\frac{x}{\alpha_{1}}\right) \hat{\delta}\left(\frac{\alpha_{2}}{p}\right) .
$$

Hence we get for $f$ and $g$ as before

$$
\begin{aligned}
\Gamma_{2} \sim f * g & =-c(q, p, x) \sum a_{k \ell} x^{k} p^{\ell} b_{\gamma \beta}\left(x q^{-\ell}\right)^{\gamma}\left(p q^{k}\right)^{\beta} \\
& =c \sum a_{k l} b_{\gamma \beta} x^{k+\alpha} p^{\ell+\beta} q^{k \beta-\ell \gamma}
\end{aligned}
$$

leading to the following proposition.

Proposition 4.1. For $f(x, p)=\sum a_{k \ell} x^{k} p^{\ell}$ and $g(x, p)=\sum b_{\gamma, \beta} x^{\gamma} p^{\beta}$ one obtains in a heuristic manner

$$
\begin{aligned}
\{f, g\}_{M} & \sim f\left(x q^{-p \partial_{p}}, p q^{x \partial_{x}}\right) g(x, p)-g\left(x q^{-p \partial_{p}}, p q^{x \partial_{x}}\right) f(x, p) \\
& \sim c(q, p, x) \sum_{k, \ell, \gamma, \beta} a_{k \ell} b_{\gamma \beta} x^{k+\gamma} p^{\ell+\beta}\left(q^{k \beta-\ell \gamma}-q^{\ell \gamma-k \beta}\right),
\end{aligned}
$$

where $c(q, p, x)$ is to be stipulated (cf. Corollary 4.3 for an essentially equivalent formula). Note by inspection or construction $\{f, g\}_{M}=-\{g, f\}_{M}$, and (4.14) appears similar to $*_{W}^{q}$ in (3.12).

If we use the formulation of (2.19), (2.20), (2.21), (2.22), and (2.23) a slightly different formula emerges involving a multiplicative factor which is missed by the analogy constructions above. Thus we check the passage (2.19) to (2.23). Equation (2.19) is the same as (2.12) corresponding to (4.3) and (2.20) corresponds to

$$
v_{q}[f](x, p,-\mathbf{a}) \sim c \sum_{r, s} q^{m s-n r} f\left(q^{-r} x, q^{-s} p\right)
$$

which would follow from (4.3) by sending $(m, n) \rightarrow-(m, n)$ and $(r, s) \rightarrow-(r, s)$. This makes sense if the sums are $-\infty \rightarrow \infty$ and there seems to be no objection to that. Then 
one would have (taking now $\nabla_{\mathbf{a}} \sim \check{\nabla}_{m n}$ as in (3.2))

$$
\begin{gathered}
X_{f}^{D}=\int d \mathbf{a} v_{\lambda}[f](\mathbf{x}, \mathbf{a}) \nabla_{\mathbf{a}} \sim c \sum_{m, n} v_{q}[f](x, p, m, n) \check{\nabla}_{m n} \\
=c \sum_{m, n} \sum_{r, s} q^{m s-n r} f\left(q^{r} x, q^{s} p\right) \frac{q^{m x \partial_{x}} q^{n p \partial_{p}}-q^{-m x \partial_{x}} q^{-n p \partial_{p}}}{\left(q^{m}-q^{-m}\right)\left(q^{n}-q^{-n}\right) x p}, \\
X_{f}^{D}=-\int d \mathbf{a} v_{\lambda}[f](\mathbf{x},-\mathbf{a}) \nabla_{\mathbf{a}} \sim X_{f}^{D}=c \sum_{m, n} v_{q}[f](x, p,-m,-n) \check{\nabla}_{-m,-n} \\
=c \sum_{m, n, r, s} q^{m s-n r} f\left(q^{-r} x, q^{-s} p\right) \frac{q^{-m x \partial x} q^{-n p \partial_{p}}-q^{m x \partial_{x}} q^{n p \partial_{p}}}{\left(q^{-m}-q^{m}\right)\left(q^{-n}-q^{n}\right) x p} \\
=-c \sum_{m, n, r, s} q^{m s-n r} f\left(q^{-r} x, q^{-s} p\right) \frac{q^{m x \partial_{x}} q^{n p \partial_{p}}-q^{-m x \partial_{x}} q^{-n p \partial_{p}}}{\left(q^{m}-q^{-m}\right)\left(q^{n}-q^{-n}\right) x p}
\end{gathered}
$$

exactly as in (2.21) (note the minus sign appears in the last equation instead of at the beginning). Hence

$$
\begin{aligned}
X_{f}^{D} & =\frac{1}{2}((4.16)+(4.17))=c \sum_{m, n, r, s} q^{m s-n r}\left[f\left(q^{r} x, q^{s} p\right)-f\left(q^{-r} x, q^{-s} p\right)\right] \check{\nabla}_{m n} \\
& =c \sum_{m, n, r, s} q^{m s-n r}\left(q^{r}-q^{-r}\right)\left(q^{s}-q^{-s}\right) x p \check{\nabla}_{r s} f \check{\nabla}_{m n}
\end{aligned}
$$

which is a difference version of (2.22). One sees that factors of $\left(q^{r}-q^{-r}\right),\left(q^{s}-q^{-s}\right)$, $\left(q^{m}-q^{-m}\right)$, and $\left(q^{n}-q^{-n}\right)$ have become involved in place of powers of $\lambda$ and this must be clarified; otherwise the patterns go over.

To clarify we compare (4.4) and (4.18) and write (4.18) in the form

$$
\begin{aligned}
{ }_{1} X_{f}^{D} g=\frac{c}{x p} \sum & q^{m s-n r}\left[f\left(q^{r} x, q^{s} p\right)-f\left(q^{-r} x, q^{-s} p\right)\right] G(q, m, n) \\
& \times\left[g\left(x q^{m}, p q^{n}\right)-g\left(x q^{-m}, p q^{-n}\right)\right]
\end{aligned}
$$

where $G^{-1}(q, m, n)=\left(q^{m}-q^{-m}\right)\left(q^{n}-q^{-n}\right)=G(q,-m,-n)$. Set $f_{ \pm} \sim f\left(q^{ \pm m} x, q^{ \pm n} p\right)$ so in an obvious notation

$$
{ }_{1} X_{f}^{D} \mathfrak{g}=\frac{c}{x p} \sum q^{m s-n r} G(q, m, n)\left[f_{+} g_{+}+f_{-} g_{-}-f_{+} g_{-}-f_{-} g_{+}\right]
$$

Now evidently, changing $m, n \rightarrow-m,-n$, one obtains a formula $\sum q^{m s-n r} G(q, m, n) f_{+} g_{-}$ $\rightarrow \sum e^{-m s+n r} G(q, m, n) f_{-} g_{+}$, and so forth, so

$$
{ }_{1} X_{f}^{D} \mathfrak{g}=\frac{c}{x p} \sum q^{-m s+n r}\left(f_{+} g_{-}+f_{-} g_{+}-f_{+} g_{+}-f_{-} g_{-}\right)
$$

leading to

$$
\begin{aligned}
&{ }_{1} X_{f}^{D} g=c x p \sum G(q, m, n)\left(q^{m s-n r}-q^{-m s+n r}\right)\left[f\left(q^{r} x, q^{s} p\right)-f\left(q^{-r} x, q^{-s} p\right)\right] \\
& \times\left[g\left(x q^{m}, p q^{n}\right)-g\left(x q^{-m}, p q^{-n}\right)\right] .
\end{aligned}
$$


This is similar to (4.4) which has the form

$$
\begin{aligned}
{ }_{2} X_{f}^{E} \mathcal{g} & =\hat{c} \sum\left(q^{m s-n r}-q^{-m s+n r}\right) f_{+} g_{+} \\
& =\hat{c} \sum() f_{-} g_{-}=-\hat{c} \sum() f_{+} g_{-}=-\hat{c} \sum() f_{-} g_{+}
\end{aligned}
$$

which implies

$$
\begin{aligned}
&{ }_{2} X_{f}^{D} g=\hat{c} \sum\left(q^{m s-n r}-q^{-m s+n r}\right)\left[f\left(q^{r} x, q^{s} p\right)-f\left(q^{-r} x, q^{-s} p\right)\right] \\
& \times\left[g\left(x q^{m}, p q^{n}\right)-g\left(x q^{-m}, p q^{-n}\right)\right] .
\end{aligned}
$$

This is essentially the same as ${ }_{1} X_{f}^{D}$ except for the $G(q, m, n)$ factor. For esthetic reasons one prefers the form ${ }_{1} X_{f}^{D} g$ since it has the more visibly meaningful form (4.16) and $\lambda$ plays a consistent role (cf. Remark 4.4 below). Thus in summary we have the following proposition.

Proposition 4.2. The difference version of Section 2 can be expressed via

$$
\begin{aligned}
X_{f}^{D} & =\sum_{m, n} v_{q}[f](x, p, m, n) \check{\nabla}_{m n} ; \\
v_{q}[f] & =\sum_{r, s} q^{m s-n r} f\left(q^{r} s, q^{s} p\right) ; \\
\check{\nabla}_{m n} g & =\frac{g\left(x q^{m}, p q^{n}\right)-g\left(x q^{-m}, p q^{-n}\right)}{\left(q^{m}-q^{-m}\right)\left(q^{n}-q^{n}\right) x p} ; \\
X_{f}^{D} g & =\frac{1}{2 x p} \sum_{m, n, r, s} q^{m s-n r} \\
& \times \frac{\left[f\left(q^{r} x, q^{s} p\right)-f\left(q^{-r} x, q^{-s} p\right)\right]\left[g\left(q^{m} x, q^{n} p\right)-g\left(q^{-m} x, q^{-n} p\right)\right]}{\left(q^{m}-q^{-m}\right)\left(q^{n}-q^{-n}\right)} .
\end{aligned}
$$

The latter expression is our putative Moyal bracket and we have the following corollary.

COROLLARY 4.3. Writing out $X_{d}^{D} g$ for monomials $f=x^{a} p^{b}$ and $g=x^{c} p^{d}$ yields

$$
\begin{aligned}
X_{f}^{D} \mathfrak{g} & =\{f, g\}_{M} \\
& =\frac{1}{2 x p} \sum q^{m s-n r} \frac{x^{a+c} p^{b+d}\left[\left(q^{r a+b s}-q^{-r a-b s}\right)\left(q^{m c+n d}-q^{-m c-n d}\right)\right]}{\left(q^{m}-q^{-m}\right)\left(q^{n}-q^{-n}\right)} .
\end{aligned}
$$

Further since, as in (4.20), (4.21), and (4.22), one has

$$
\begin{aligned}
-\sum_{m, n} q^{m s-n r} G g_{-} & =-\sum_{m, n} q^{-m s+n r} G g_{+}, \\
-\sum_{r, s} q^{m s-n r} f_{-} & =-\sum q^{-m s+n r} f_{+}
\end{aligned}
$$

there results

$$
X_{f}^{D} g=\frac{1}{2 x p} \sum \frac{q^{m s-n r}-q^{-m s+n r}}{\left(q^{m}-q^{-m}\right)\left(q^{n}-q^{-n}\right)} f\left(q^{r} x, q^{s} p\right) g\left(q^{m} x, q^{n} p\right) .
$$


This is reminiscent of (4.4) but with a $G(q, m, n)$ factor so the calculation (4.14) applies with $G(q, m, n)$ inserted and consequently $\{f, g\}_{M}=-\{g, f\}_{M}$ as before, although this is not immediately visible from (4.28). We note also from (4.25) or (4.28) that it does no harm to use alternatively a form based on (2.23) in the form (cf. (4.18))

$$
X_{f}^{D} g=c \sum_{m, n, r, s} q^{m s-n r} \check{\nabla}_{r s} f \check{\nabla}_{m n} \mathcal{g}
$$

which inserts an additional factor $G(q, r, s)$ into (4.25).

REMARK 4.4. The multiplicative factors involve terms $\left(q^{m}-q^{-m}\right),\left(q^{n}-q^{-n}\right)$, $\left(q^{r}-q^{-r}\right)$, or $\left(q^{s}-q^{-s}\right)$, all of which correspond to a $\lambda$ arising from $\check{\nabla}_{m n}$ or $\check{\nabla}_{r s}$; instead of coming out of the integral signs as $\lambda$ in the continuous versions of Section 2 they have to be summed. Note the correspondence $x+\lambda a_{1}=\alpha_{1}$ corresponding to $\alpha_{1} / x=q^{m}$ uses $\lambda$ in a different manner so it is at first glance surprising that ${ }_{2} X_{f}^{D}$ even comes close to ${ }_{1} X_{f}^{D}$. The relations of our formulas to the star products and Moyal brackets of (3.10), (3.11), (3.12), and (3.13) will be examined later as well as the expansion of material in [4] related to work of Curtright, Fairlie, Zachos, and the Saito school (cf. [4] for references). We note also that for a complex phase space $\{z, \zeta\}$ (not clarified) an interesting variation on the $q$-Moyal bracket of (4.14) or (4.25) is given in [18] for a KP situation (cf. also [4] where this is expanded). This is applied to a KP hierarchy context using complex variable methods and, although powers of $q$ are inserted in various places, it is not developed systematically in a $q$-analysis manner and no recourse to $q$-derivatives is indicated. We will expand further the treatment of [4] for this situation in a subsequent paper.

\section{REFERENCES}

[1] R. Carroll, Discretization, Moyal, and integrability, http://front.math.ucdavis.edu/math. QA/0105227.

[2] _ Remarks on quantum transmutation, http://front.math.ucdavis.edu/math.QA/ 0101072.

[3] _ Some applications of quantum groups and noncomutative geometry, book, in preparation.

[4] _ Quantum Theory, Deformation and Integrability, North-Holland Mathematics Studies, vol. 186, North-Holland Publishing, Amsterdam, 2000. CMP 1838460. Zbl 01544073.

[5] _ _ Various aspects of Whitham times, Acta Appl. Math. 60 (2000), no. 3, 225-316. CMP 1776 704. Zbl 992.03695.

[6] _ Star products and integrability, Proceedings 3rd Inter. Congress ISAAG, Berlin, to appear, 2001.

[7] R. W. Carroll, Topics in Soliton Theory, North-Holland Mathematics Studies, vol. 167, North-Holland Publishing, Amsterdam, 1991. MR 93f:58095. Zbl 777.35072.

[8] M. Chaichian and A. Demichev, Introduction to Quantum Groups, World Scientific Publishing, New Jersey, 1996. MR 2000a:81093. Zbl 930.17009.

[9] Ö. F. Dayi, q-deformed star products and Moyal brackets, J. Math. Phys. 39 (1998), no. 1, 489-496. MR 98k:81124. Zbl 946.53048.

[10] A. Dimakis and C. Tzanakis, Dynamical evolution in noncommutative discrete phase space and the derivation of classical kinetic equations, http://front.math.ucdavis.edu/ math-ph/9912016. 
[11] G. V. Dunne, Quantum canonical invariance-a Moyal approach, J. Phys. A 21 (1988), no. 10, 2321-2335. MR 89k:58108. Zbl 665.58049.

[12] L. D. Faddeev and L. A. Takhtajan, Hamiltonian Methods in the Theory of Solitons, Springer Series in Soviet Mathematics, Springer-Verlag, Berlin, 1987. MR 89m:58103. Zbl 632.58004.

[13] M. Fichtmüller, A. Lorek, and J. Wess, q-deformed phase space and its lattice structure, http://xxx.lanl.gov/abs/hep-th/9511106.

[14] I. Frenkel, J. Lepowsky, and A. Meurman, Vertex Operator Algebras and the Monster, Pure and Applied Mathematics, vol. 134, Academic Press, Massachusetts, 1988. MR 90h:17026. Zbl 674.17001.

[15] J. Gawrylczyk, Relationship between the Moyal KP and the Sato KP hierarchies, J. Phys. A 28 (1995), no. 15, 4381-4388. MR 97f:58067. Zbl 869.35082.

[16] A. Hebecker, S. Schreckenberg, J. Schwenk, W. Weich, and J. Wess, Representations of a qdeformed Heisenberg algebra, Z. Phys. C 64 (1994), no. 2, 355-359. MR 95k:81061.

[17] V. Kac, Vertex Algebras for Beginners, University Lecture Series, vol. 10, American Mathematical Society, Rhode Island, 1997. MR 99a:17027. Zbl 861.17017.

[18] R. Kemmoku, Difference operator approach to the Moyal quantization and its application to integrable systems, J. Phys. Soc. Japan 66 (1997), no. 1, 51-59. MR 98i:58107. Zbl 945.37013.

[19] R. Kemmoku and S. Saito, Phase space discretization and Moyal quantization, http://arxiv. org/abs/hep-th/9510077.

[20] _ Discretization of Virasoro algebra, Phys. Lett. B 319 (1993), no. 4, 471-477. MR 95b:81075.

[21] _ Difference operator approach to the Moyal quantization, J. Phys. Soc. Japan 65 (1996), no. 7, 1881-1884. MR 97j:81172. Zbl 942.81583.

[22] _ $\quad W_{1+\infty}$ as a discretization of Virasoro algebra, J. Phys. A 29 (1996), no. 14, 41414148. MR 97j:81109. Zbl 899.17014.

[23] M. Klimek, Extension of q-deformed analysis and q-deformed models of classical mechanics, J. Phys. A 26 (1993), no. 4, 955-967. MR 94c:81077. Zbl 772.70014.

[24] A. Lorek, A. Ruffing, and J. Wess, A q-deformation of the harmonic oscillator, http://arxiv. org/abs/hep-th/9605161.

[25] J. Schwenk, q-deformed Fourier theory, http://arxiv.org/abs/hep-th/9406168.

[26] J. Schwenk and J. Wess, A q-deformed quantum mechanical toy model, Phys. Lett. B 291 (1992), no. 3, 273-277. MR 93h:81066.

[27] Y. Smirnov and A. Turbiner, Hidden $s_{2}$-algebra of finite-difference equations, http://arxiv. org/abs/funct-an/9512002.

[28] _ Lie-algebraic discretization of differential equations, http://arxiv.org/abs/functan/9501001.

[29] I. A. B. Strachan, The dispersive self-dual Einstein equations and the Toda lattice, http:// arxiv.org/abs/hep-th/9606101.

[30] _ The Moyal bracket and the dispersionless limit of the KP hierarchy, J. Phys. A 28 (1995), no. 7, 1967-1975. MR 96b:58065. Zbl 829.35116.

[31] _ A geometry for multidimensional integrable systems, J. Geom. Phys. 21 (1997), no. 3, 255-278. MR 98b:58066. Zbl 893.58061.

[32] A. Turbiner, Canonical discretization. I. Discrete faces of (an)harmonic oscillator, http:// arxiv.org/abs/hep-th/0004175.

[33] J. Wess, q-deformed Heisenberg algebras, Geometry and Quantum Physics (Schladming, 1999), Lecture Notes in Phys., vol. 543, Springer, Berlin, 2000, pp. 311-382. MR 2001g:81127.

Robert CARroll: Department of MAthematics, University of Illinois, Urbana, IL 61801, USA

E-mail address: rcarro11@math. uiuc. edu 


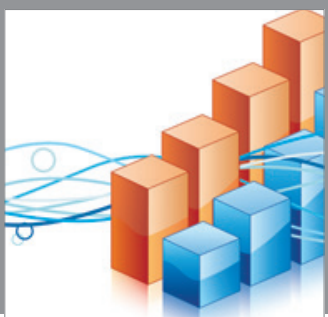

Advances in

Operations Research

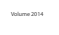

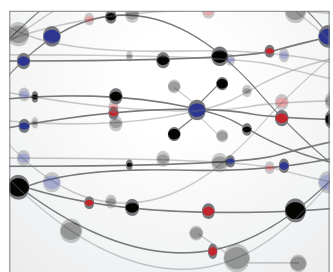

\section{The Scientific} World Journal
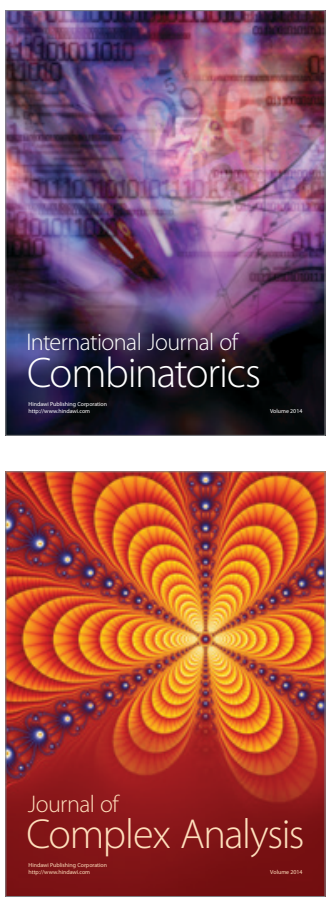

International Journal of

Mathematics and

Mathematical

Sciences
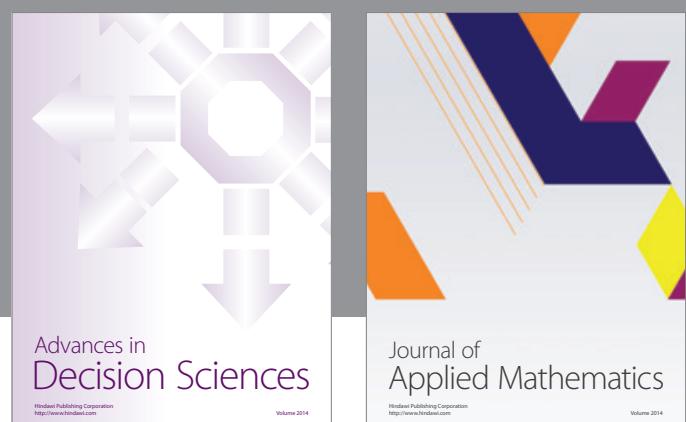

Journal of

Applied Mathematics
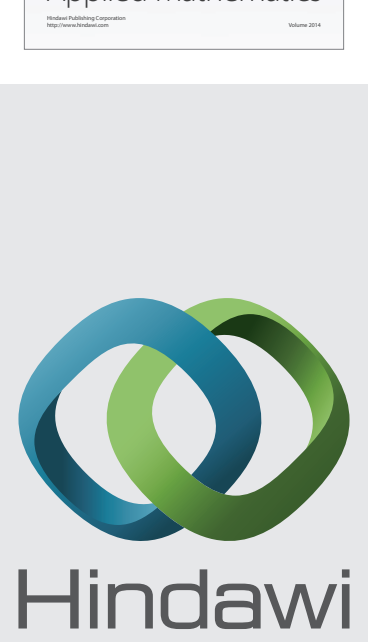

Submit your manuscripts at http://www.hindawi.com


Mathematical Problems in Engineering
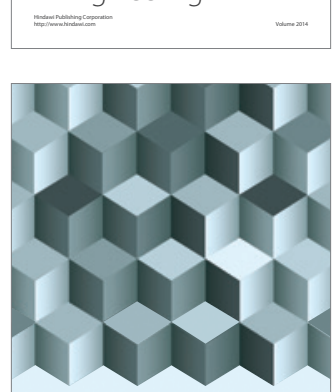

Journal of

Function Spaces
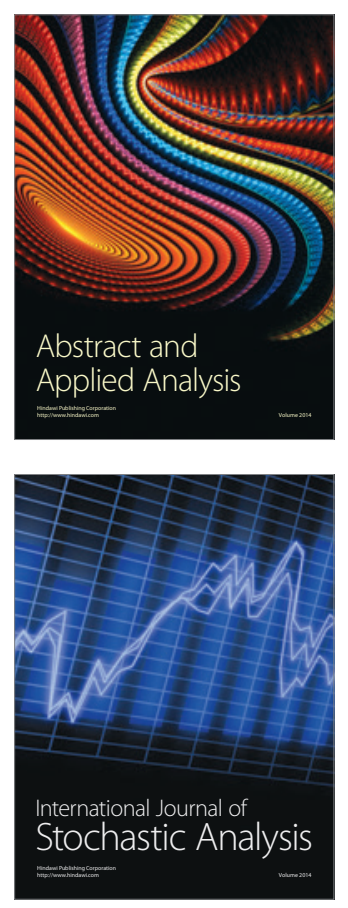

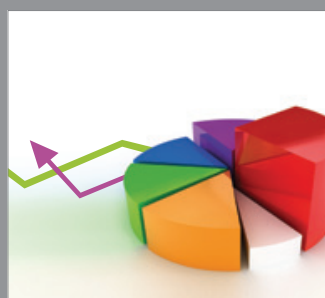

ournal of

Probability and Statistics

Promensencen
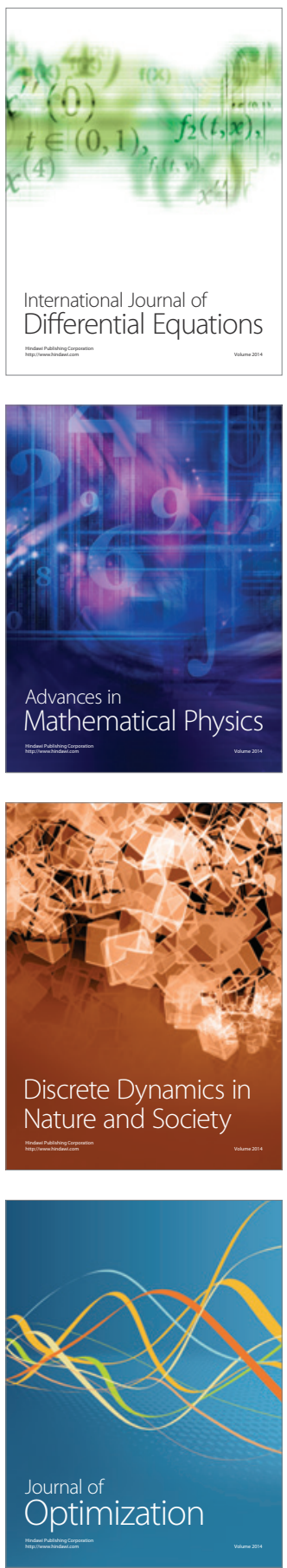